\title{
Implementation of Zone Routing Protocol for Heterogeneous Hybrid Cluster Routing to Support QoS in Mobile Ad hoc Networks
}

\author{
B.Sreedevi \\ Srinivasa Ramanujan \\ Centre, SASTRA University, \\ Kumbakonam, \\ Tamil Nadu, India
}

\author{
Dr.Y.Venkataramani \\ Saranathan College of \\ Engineering, Trichy, \\ Tamil Nadu, India.
}

\author{
Dr.T.R.Sivaramakrishnan \\ SASTRA University, Tanjavur, \\ Tamil Nadu, India.
}

\begin{abstract}
The dynamic nature of mobile ad hoc networks (MANETs) is attributed to several gateway selection schemes have been proposed that select gateway nodes based on single QoS metric, for instance link availability, link capacity etc. or Hybrid cluster routing metrics, such as the combination of gateway node speed, residual energy, and number of hops, for mobile ad hoc networks (MANETs). The nodes build the routing table statically as well as on-demand and therefore they exhibit hybrid nature in routing. The nodes are grouped together called cluster and each group is monitored by a cluster head during data transmission. The routing is performed either by inter-clustering where the source node and destination node belongs to different clusters or by intra-clustering where the source node and destination node belong to same cluster. In this paper, we have introduced a new term called Partial Authority Node (PAN) which shares the load form cluster head and thereby performs intra-cluster routing efficiently. Also a gateway node or a border node $(\mathrm{BN})$ is available for inter-cluster routing to trace the destination easily. So, most of the load is shared by PAN and BN. The algorithm is evaluated using Zone Routing Protocol by making simulations in ns2 and the results shows the performance in terms of throughput, packet delivery ratio, and lowered delay and hence it provides a better quality of service.
\end{abstract}

\section{Keywords}

QoS Metrics, ad hoc network, gateway selection, end-to-end QoS metrics, end-to-end delay, Energy consumption, heterogeneous cluster, Quality of Service (QoS),ZRP.

\section{INTRODUCTION}

QoS routing is an important component of such networks. The objective of QoS routing is two-fold: to find a feasible path for each transaction; and to optimize the usage of the network by balancing the load. Routing in mobile ad-hoc network depends on many factors like, including modeling of the topology, choice of routers, and initiation of request, and specific underlying characteristics that could serve as a heuristic in finding the path efficiently. The routing problem in mobile ad-hoc networks relates to how mobile nodes can communicate with one another, over the wireless media, without any support from infrastructure network components. Several routing algorithms have been proposed in the literature for mobile ad-hoc networks with the goal of achieving efficient routing. Proactive protocols perform routing operations between all source destination pairs periodically, irrespective of the need of such routes where as Reactive protocols are designed to play down routing overhead. Instead of tracking the changes in the network topology to continuously maintain shortest path routes to all destinations, Reactive protocols determine routes only when essential. Hybrid Routing is an approach that is often used to win a better balance between the adaptability to varying network conditions and the routing overhead. These protocols utilize a combination of reactive and proactive principles, each applied under different conditions, places, or regions.

Most existing routing protocols assume homogeneous mobile ad hoc networks (MANETs), that is, all the nodes in the network have the same transmission power (range), transmission data rate, processing capability, reliability and security level. However, a homogeneous ad hoc network suffer from poor scalability i.e., the network performance is degraded quickly as the number of nodes increases, which has been demonstrated by theoretical analyses, simulation experiments and testbed measurements [5]. Furthermore, in many realistic ad hoc networks, nodes are actually heterogeneous [6] [7] [8]. For example, in a battle field network, portable wireless devices are carried by soldiers and more powerful and reliable communication devices are carried by vehicles, tanks, aircraft and satellites; these devices/ nodes have different communication characteristics in terms of transmission power, data rate, processing capability, reliability, etc. so it would be more realistic to model these network elements as different types of nodes [3].

Creating and maintaining distributed network structures like dominating sets, clusters, spanning graph, etc has been the commonly agreed upon solution for organizing wireless networks in a large scale topology. This would help to enable efficient, reliable and prolonged communication and cooperation between nodes in a dynamic environment. Among these, clustering is a most familiar approach [9]. Routing is performed by node disjoint virtual circuit approach incurs some delay [15]. End to end security is made in routing to ensure reliability [16]. Clustering provides for efficient radio resources allocation, location and energy management, routing and backbone formation in ad hoc wireless networks and is an efficient method for tackling the scalability issues pertinent to this networking domain [10]. In wireless networks, in which possibly all nodes can be mobile, partitioning the nodes into groups (clusters) is 
similarly important [1]. Clustering may be used for controlling spatial reuse of the shared channel (e.g., in terms of time division or frequency division schemes), for minimizing the amount of data to be exchanged in order to maintain routing and control information in a mobile environment, as well as for building and maintaining cluster based virtual network architecture.

In existing solutions for clustering ad hoc networks, clustering is performed in two phases: clustering setup and clustering maintenance. The first phase is accomplished by choosing some nodes that act as coordinates of the clustering process (cluster heads). Then a cluster is formed by associating a cluster head with some of its neighbors (i.e., nodes within the cluster head's transmission range) that become the ordinary nodes of the cluster [1]. In cluster based topology, there are two types of flows. They are inter-cluster and intra-cluster flows [9]. The intra-cluster flows originate and terminate in the same cluster, whereas, the inter-cluster flows are meant for communication between clusters. The cluster structure imposes many important restrictions and difficulties in the calculation / estimation, transfer / exchange and updates of link state information those are inevitable for QoS implementations. Thus, traditional QoS metrics like bandwidth, delay, etc can never be accurately estimated or predicted for inter-cluster paths even though certain level of accuracy can be guaranteed in the case of intra-cluster flows. There are not many works that tackles QoS routing issues in the clustered topology, except those presented in [11] [12].

\section{HYBRID ADHOC ROUTING PROTOCOLS}

This section introduces a hybrid model that combines reactive and proactive routing protocols but also a location assisted routing protocol. The Zone Routing Protocol (ZRP) is a hybrid routing protocol that divides the network into zones. The IntraZone Routing Protocol (IZRP) implements the routing within the zone, while the Inter-zone Routing Protocol (IERP) implements the routing between zones. ZRP provides a hierarchical architecture where each node has to maintain additional topological information requiring extra memory. The Location Aided Routing (LAR) [42] is a location assisted routing protocol that uses location information for the routing functionality.

\subsection{Our Approach}

In this work, we focus Heterogeneous hybrid Cluster Routing to Support QoS in Mobile Ad hoc Networks. This algorithm tries to achieve performs intra-cluster routing efficiently. Here each of these objectives depends upon Heterogeneous hybrid Cluster Routing parameters. Depending upon the parameters we are considering and usage, different objectives can be achieved. These parameters can be varied depending upon the application. As every application have different requirements of the QOS and thus have different priorities of each parameter. So we have proposed a flexible generic scheme in which user can select different set of QOS parameters accounting for achieving multiple objectives.

We also presents clustering on heterogeneous mobile ad hoc networks with the new term Partial Authority Node (PAN) has been introduced and discussed. The algorithm differs from others by the introduction of PAN [9]. It consists of two phases:
In the first phase all the nodes are grouped into clusters with each cluster head $(\mathrm{CH})$ and a gateway. Then PAN are selected for each node for each group (cluster).In the second phase, routing is performed where the source finds its destination either by inter-cluster routing if they belong to different clusters or by intra-cluster routing if they belong to the same cluster.

\subsection{Neighbor Discovery Phase}

Here Neighbor Discovery Algorithm will look after the maintenance of Neighbor Tables and Zone Routing tables. Each and every node maintains Neighbor Tables and Zone Routing Tables. The Neighbor Table along with the neighbor node addresses too stores available QOS parameter values along the link between itself and its Neighbor. These parameters are considered for selecting best available routes by Intra Zone Routing Protocol (used to choose the routes with in the zone). In this phase each and every node periodically transmits beacons to its neighbors. On reception of these packets from neighbors every node updates its Neighbor Table with appropriate values. Each node exchanges their Neighbor Tables from their corresponding neighbors and constructs Zone Routing Tables.

\subsection{Route Selection Phase}

Multi Objective Optimization is used in Route Selection Phase, where Multi Objective Problem is transformed in to Single Objective Problem by weighting route. The goal of such singleobjective optimization problems is to find the best solution, which corresponds to the minimum or maximum value of an objective function. In this algorithm multiple objectives are reformulated as single-objective problem by combining different objectives into one (that is, by forming a weighted combination of the different objectives). First, all the objectives need to be either minimized or maximized. This is done by multiplying one of them by -1 (i.e., $\max f 2$ is equivalent to $\min (-\mathrm{f} 2)=\operatorname{minf} 2$ '). Next, these objectives must be lumped together to create a single objective function. Weighting (conversion) factors w $1 \mathrm{w} 2 \ldots$ wn are used in order to win a single, combined objective function.

Maximize $\{\mathrm{F}\}=(+/-) \mathrm{w} 1 \mathrm{f} 1(\mathrm{x})+(+/-) \mathrm{w} 2 \mathrm{f} 2(\mathrm{x}) \ldots(+/-) \mathrm{wnfn}(\mathrm{x})$.

To find the relative performance of each objective function each of the objective function value obtained is divided by corresponding desired QOS value. Now relative efficiency of each route is obtained by calculating the $F$ value of all effective paths (which satisfy the QOS requirements) from source to destination. Finally, given this single objective function one can find a single optimal solution (optimal route).

\subsection{Route Establishment Phase}

This phase is for reverse path set up i.e. the route is established from destination to source. After selecting the optimal route available by Route Selection phase, Route Reply packets will be sent along the path selected, back tracking from destination to source setting the status field value of corresponding entry in route table from Route Not Established (NE) to Established, and updating Next Node_ID as the P.Current_ID (the node from where RREP packet has received). Then send back the RREP packets towards source selecting next hop from route table which is stored during push path set up. 
We present a new heterogeneous cluster based algorithm which provides support for QoS in terms of throughput, packet delivery ratio (PDR) and delay for mobile ad hoc networks. In this algorithm, routing is performed in two phases.

\section{INTER-CLUSTER ROUTING AND INTRA-CLUSTER ROUTING}

We have introduced a term called Partial Authority Node (PAN) for intra-cluster routing, which assists cluster head and takes care of all work for routing by itself whereas, in inter-clustering, a border $(\mathrm{BN})$ or a gateway is introduced, so as to trace the destination cluster.

\subsection{Intra-cluster routing}

Initially all the nodes are put to "none" state. After initialization, depending upon its responsibility, $\mathrm{CH}$ and $\mathrm{MN}$ are determined. Each node finds its immediate neighbors i.e., one hop neighbor and builds a routing table. At this moment, few PAN's are determined. Each node calculates its distance to all PAN. So, the distance between all nodes and PAN's are calculated. The MN with least distance to PAN is associated. Finally, all the MN gets associated with one $\mathrm{CH}$ and a PAN per each cluster. Now, any node may act as a source (S) and may forward the data packets to the destination node (D). If the destination node is within the cluster, intra-clustering is performed.

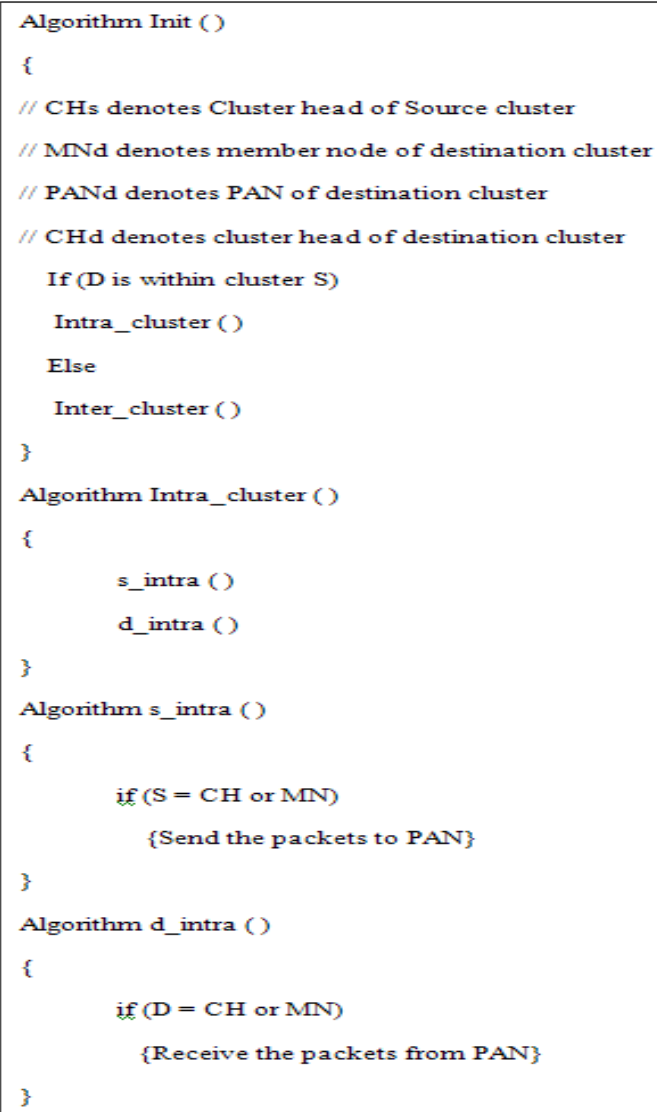

The algorithm is briefed as follows: If the $\mathrm{S}$ is $\mathrm{CH}$ or a $\mathrm{MN}$, then data packets are delivered to PAN and PAN will further deliver to D. Else if S is PAN, then S will directly deliver to D. In the case of Intra-cluster, all the responsibilities will be by PAN, rather than by $\mathrm{CH}$. $\mathrm{CH}$ will be engaged in inter-cluster routing. In order to avoid the burden of $\mathrm{CH}, \mathrm{PAN}$ is introduced and setup and it shares the $\mathrm{CH}^{\text {'s }}$ work in case of intra-clustering. The selection of PAN by each node to associate is done during initialization and it is build along with the routing table.

\subsection{Inter-cluster routing}

Inter-cluster routing is performed when the source $S$ and destination $\mathrm{D}$ are not in the same cluster. The routing is performed and is shown in the algorithm as below: This algorithm is performed in three stages and is explained as follows: The first stage is at Source cluster, where the source checks its status and finally forwards the packet to cluster head accordingly. In the second stage, the cluster head of source delivers the packets to gateway and thereon it is forwarded to the cluster head of destination. In the third stage, the cluster head delivers the packet to final target according to its status and the details of delivery have been discussed in the algorithm. In Inter-clustering $\mathrm{BN}$ plays an important role by tracing out the target (cluster), whereas, within the cluster PAN plays a vital role by sharing the work of cluster head and thereby reduces the traffic at $\mathrm{CH}$. So, $\mathrm{CH}$ performs better and keeps monitoring the entire routing process by sharing its work with PAN and BN.

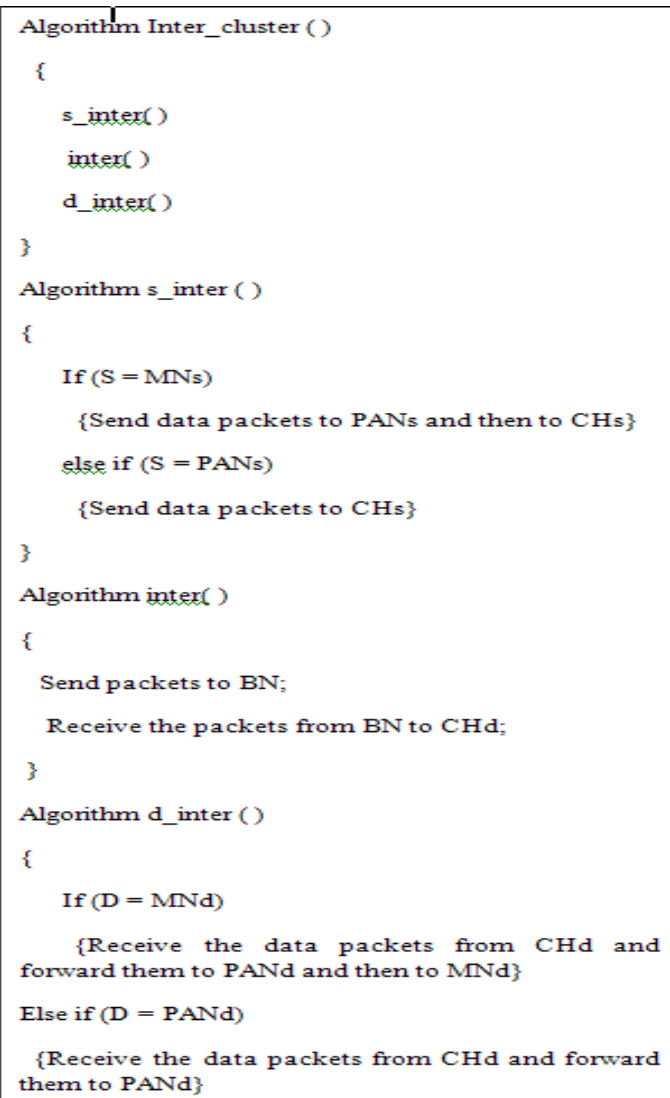


In the best case the required route is directly found from the routing table in one step, i.e. in $\mathrm{O}(1)$ time. So, the best case time complexity of the algorithm for the complete network is

$\mathrm{N} *(\mathrm{O}(1)+\mathrm{O}(\mathrm{n} 2))=\mathrm{O}(\mathrm{N} * \mathrm{n} 2)$

\section{SIMULATION PARAMETERS}

We use a detailed simulation model base on ns2 [13][14]. The DCF of IEEE 802.11 for Wireless LAN is used as the MAC Layer [14]. The radio model uses characteristics similar to Lucent's Random waypoint mobility model to model node movements. The simulation time is 10 seconds. We consider network size with 24 nodes in a rectangular field of $400 \times 600$ with the speed varying from 0 to maximum. Traffic pattern is CBR / UDP connection. The radio propagation range is about 64 $\mathrm{m}$. Data packets have a fixed size of 512 bytes with the data rate of $1 \mathrm{Mb} / \mathrm{s}$ and has the maximum of 1000 packets with the queue length of 10 .

\section{SIMULATION RESULTS}

Several comparisons are made on the intra-clustered routing and inter-cluster routing using zone routing protocol (ZRP) for delay, throughput and PDR. The discussions about their performance are given below.

\subsection{Intra-cluster Routing}

\subsubsection{Delay}

The result on delay shows (Figure.1) that ZRP keeps delay with variations. $\mathrm{X}$ axis shows the time scale in micro seconds and $\mathrm{Y}$ axis as its delay. The delay variations are due to searching of PAN and $\mathrm{CH}$ and exchanging of routing information.

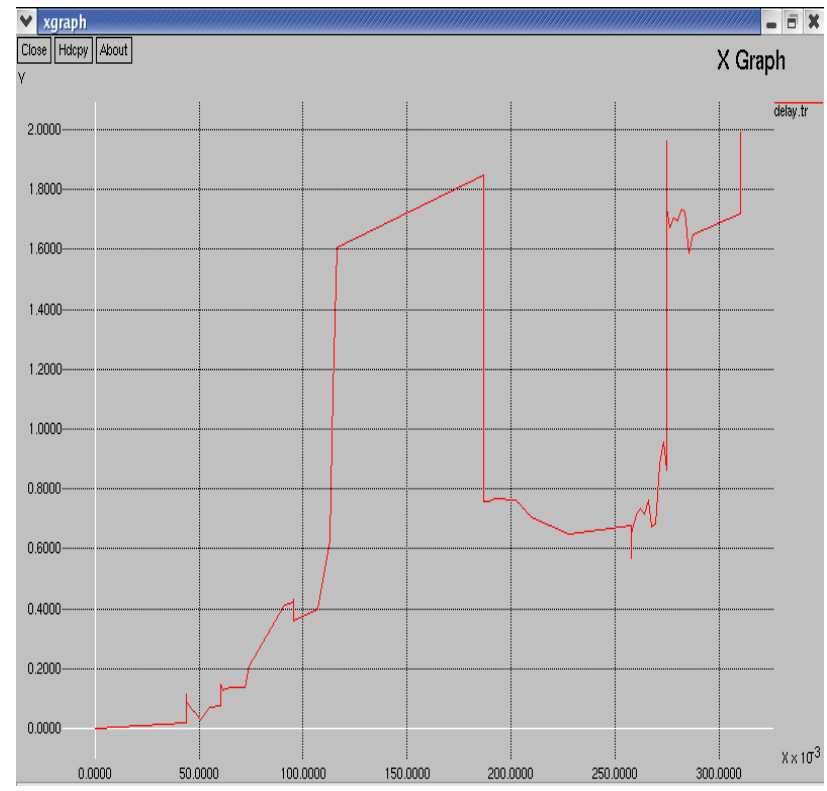

Figure 1. Delay

\subsubsection{Packet Delivery Ratio (PDR)}

The result of PDR shows (figure.2) that PDR reaches higher value and then goes down slightly as it performs searching.

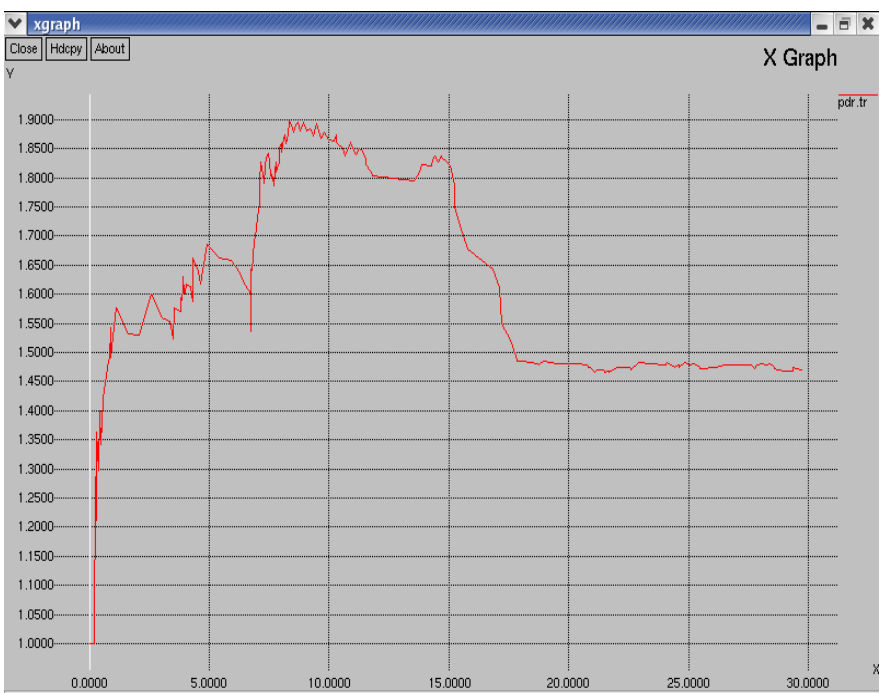

Figure 2. Packet Delivery Ratio

\subsubsection{Throughput}

The results of throughput (figure. 3) shows that it increases drastically and suddenly decreases it takes some delay in finding out its own PAN and $\mathrm{CH}$.

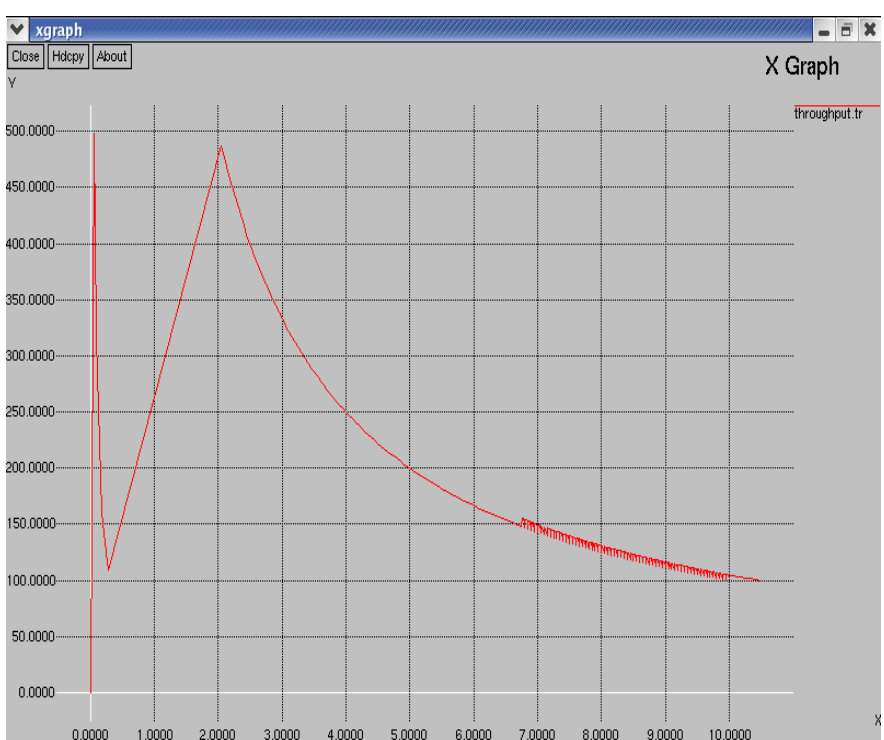

Figure 3. Throughput

\subsection{Inter-Cluster routing}

\subsubsection{Delay}

The result on delay shows (Figure .4) that ZRP keeps delay with variations. $\mathrm{X}$ axis shows the time scale in micro seconds and $\mathrm{Y}$ axis as its delay. The delay variations are due to searching of $\mathrm{PAN}$ and $\mathrm{CH}$ and exchanging of routing information among $\mathrm{BN}$ and $\mathrm{CH}$. 


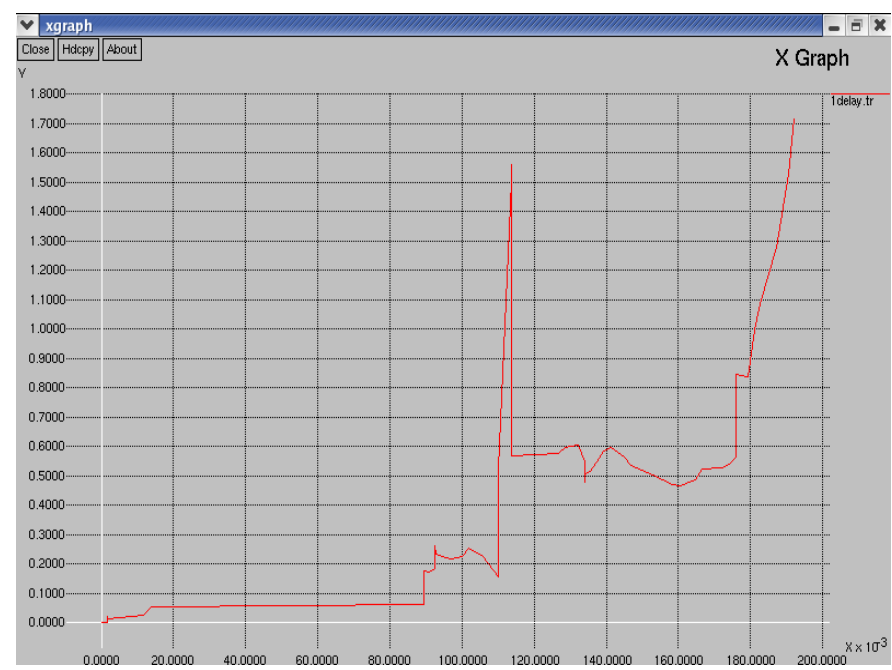

Figure 4 . Delay in inter-cluster routing

\subsubsection{Packet Delivery Ratio (PDR)}

The result of PDR shows (figure 5) that PDR reaches higher value and then goes down slightly as it performs searching of $\mathrm{BN}, \mathrm{CH}$ and PAN.

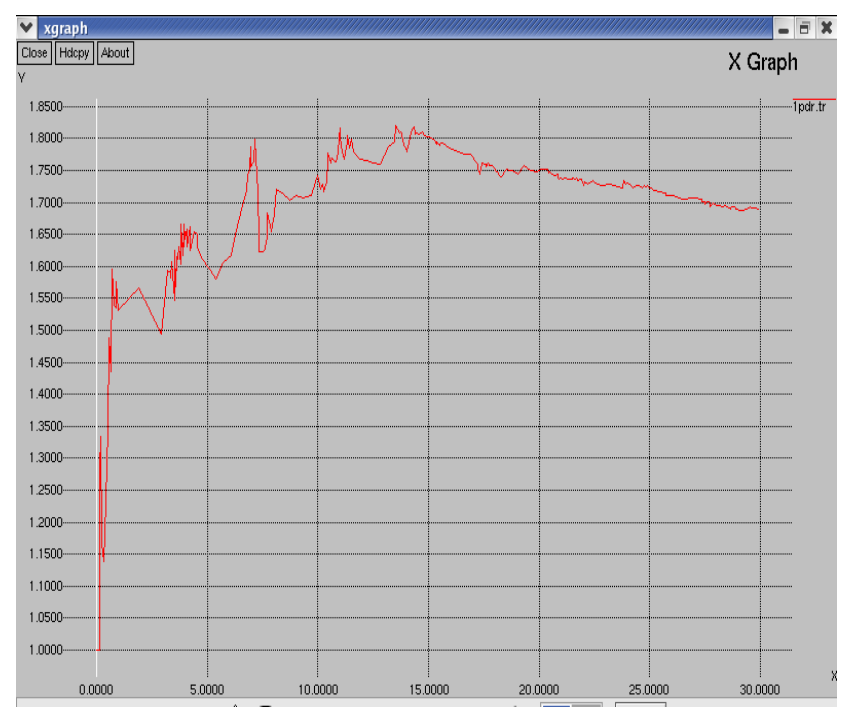

Figure 5 . PDR in Inter- cluster routing

\subsubsection{Throughput}

The results of throughput (figure.6) show that it increases drastically and abruptly decreases as it takes some delay in finding out its own PAN and $\mathrm{CH}$ and between $\mathrm{BN}$ and $\mathrm{CH}$.

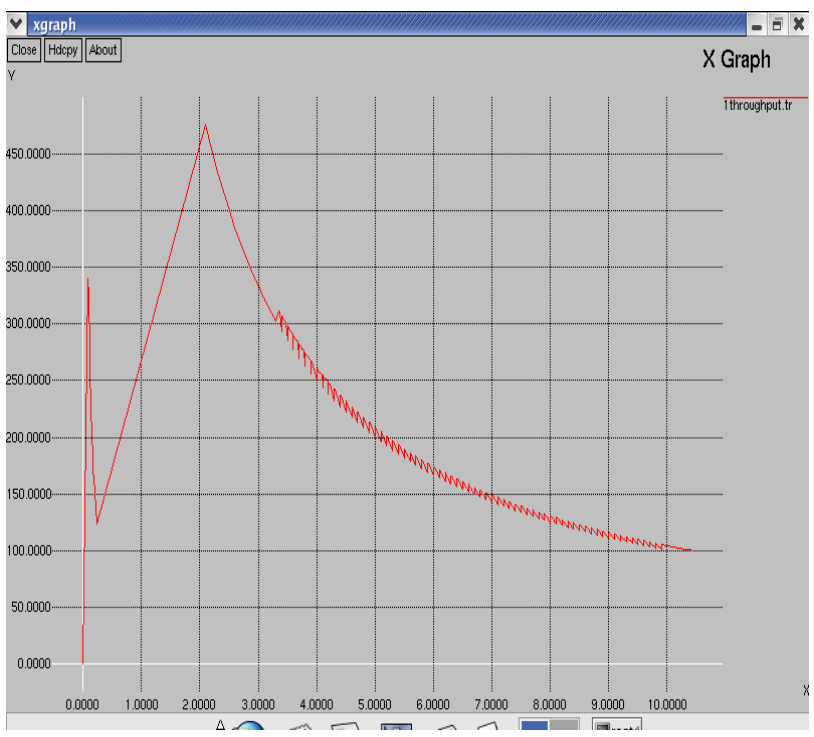

Figure 6. PDR in Inter cluster routing.

\section{CONCLUSIONS \& FUTURE WORK}

In this work, we have shown that the throughput reaches hike and thereby, produces a good output for both intra-cluster and inter-cluster routing. There is no metric proposed for selecting cluster head and partial authority node as far as this paper is concerned. In our future work this would be important criteria for selecting such nodes. The QoS parameters are (min) delay, PDR and (max) throughput are taken into account for the present paper. In future, some more parameters like transmission power, Connection resilience, residual energy and priority of packets could be taken for consideration.

\section{REFERENCES}

[1] S.Basagni. "Distributed and mobility-adaptive clustering for Ad hoc networks". Technical Report UTD/EE-02-98, Erik Jonsson School of Engineering and Computer Science, The University of Texas at Dallas, July 1998.

[2] Puneet Sethi, Gautam Barua, "C RESQ: Providing QoS and Security in Ad hoc Networks", Proceedings of 11th Euromicro Conference on Parallel, Distributed and Network-Based Processing (Euro-PDP'03), IEEE,2003.

[3] Xiaojiang (James) Du, Depeng Wu, Wei Lu, Yuguang Fang, "Multiclass Routing and Medium Access Control for Heterogeneous Mobile Ad Hoc Networks", IEEE Transactions on Vehicular Technology, Vol.55, No.1.January 2006.

[4] Tinku Rasheed, Khaldoun Al Agha, Usman Javaid, Laurent Reynoud, "Cluster-Quality based Hybrid Routing Large Scale Mobile Multi-hop Networks", IEEE Communications Society 2007.

[5] K.Xu, X.Hong and M.Gerla, "An ad hoc network with mobile backbones," in Proc.IEEE ICC 2002, Vol.5, NewYork, NY, April 2002. pp 3138-3143.

[6] Y.Yang and R.Kravets, "Achieving Delay Guarantees in Ad Hoc Networks through Dynamic Contention Window Adaptation", in proc. IEEE INFOCOM 2006. 
[7] IEEE Computer Society, "802.11: Wreless LAN Medium Access Control (MAC) and Physical Layer (PHY) Specifications"

[8] G.Pei, M.Gerla, X.Hong and C.C. Chiang, "A Wireless Hierarchical Routing Protocol with Group Mobility," in Proc. IEEE Wireless Communication and Networking Conference WCNC, pp 1536-1540,1999.

[9] J.Y.Yu and P.H.J Chong, "A Survey of Clustering Schemes for Mobile Ad hoc Networks", IEEE Commun, Surveys and Tutorials, vol.7, No.1.pp 32-48,Q1,2005.

[10] T.M Rasheed, L.Reynaud, U.Javaid and K.Al.Agha, “An Efficient Stable Clsutering Algorithm for Scalable Mobile Multi-hop Networks", IEEE CCNC 2007.

[11] R.Ramanathan and M.Steenstrup ," HierarchicallyOrganized Multihop Mobile Wireless Networks for Quality -of- Service Support", ACM Journal on Mobile Networks and Applications, vol.3,No.1,pp.101-119,1998.

[12] J.Warland et al ," Adaptive Quality-of-Service for Mobile Ad hoc Network," in Proc. of International Conference on
Mobile Wireless Communications Networks (MWCN'03), Singapore,October,2003.

[13] K. Fall and K. Varadhan (Eds.). "ns notes and documentation", $1999 . \quad$ Available from http://www.mash.cs.berkeley.edu/ns/

[14] NS2: Network Simulator: http://www.isi.edu/nsnam/ns/.

[15] Sreedevi. B, Venkataramani .Y, Sivaramakrishnan T.R., "Node Disjoint-Virtual Circuit Approach in Ad Hoc OnDemand Multipath Distance Vector Routing to offer Quality of Service in Ad Hoc Networks", International Journal of Engineering Science and Technology(IJEST ), ISSN : 0975-5462, April 2011, Vol. 3 issue 4, pp.3045 3050 .

[16] Sreedevi. B, Venkataramani .Y, Sivaramakrishnan T.R.," Implementing End-To-End Reliability and Energy Conservation Routing to Provide Quality of Service in Mobile Ad hoc Networks", European Journal of Scientific Research (EJSR), ISSN 1450-216X, Vol.55 No.1 (2011), pp.28-36 